\title{
Who Is Hikonyan? The Phenomenon of Japanese Yuru-Chara*
}

\author{
Jillian Rae Sutera
}

\begin{abstract}
In 2007, Hikonyan was the first winner of the Japan-wide "yuru-chara" or "yurui mascot character" competition. Since then, yuru-chara, or unsophisticated characters, have become a part of the mascot character marketing industry worth over a billion Japanese yen. However, they have not expanded far outside of Japan. This paper looks at yuru-chara through articles, documentation, literature, and interviews to explore their history and status in Japan, and attempts to explain their rapid rise within the country as well as their lack of presence outside of the country. These resources help explain the design choices of yuru-chara, the reasoning behind those choices, and what they mean within Japan's society. They additionally explain how yuru-chara impact the Japanese market. The paper also explores some Japanese branding and how yuru-chara can fit into the concept of the Cool Japan Project. Finally, it explores some possibilities of expanding the concept of yuru-chara to international markets and how they could successfully work abroad.
\end{abstract}

\section{Keywords}

Cool Japan, branding, authenticity, international markets, mascot characters

Hikonyan is a white mascot cat with a red and yellow "samurai" helmet. He was created in 2007, to celebrate the 400th anniversary of Hikone Castle in Hikone City, Shiga Prefecture. His name is a portmanteau of the city, Hikone and the onomatopoeia for a cat's meow (nyan). Mascot characters have been around since long before Hikonyan, but Hikonyan was different. He was the first popular "yuru-chara” as coined by Jun Miura (Miura 2005). In 2008, Hikonyan was the main character for the "Yuru-Chara Matsuri in Hikone Kigurumi Summit 2008", which would eventually become the "Yuru-Chara Grand Prix" (Yuru-Chara Matsuri in Hakone Kigurumi Summit 2008 2008). Hikonyan is the unofficial start to the yuru-chara phenomena in Japan, which has exploded in popularity since then.

Yuru-chara have become a major market player within Japan, but are still relatively unknown outside of Japan. Their strong association with local regions in
Japan and Japanese identity could be used internationally through expansion programs such as the Cool Japan Project (Cool Japan/Creative Industries Policy 2013).

The objective of this study uses the analysis of interviews, history, status, direct observations, and physical features as a framework to better understand the upward trend. The results of this paper will allow the reader to better utilize potential character management techniques.

This report is a holistic look at yuru-chara and character management within Japan's market. As such, most of the research is archival into the origins of mascot characters and yuru-chara in Japan, as well as

aKeio University, Japan

Correspondent Author:

Jillian Rae Suter, 5322 Endo, Fujisawa, Kanagawa 252-0882, Japan 
additional data on some current social media trends. This research was taken mostly from secondary sources, including company profile pages, research books, blogs, online videos, government pages, store merchandising, and pamphlets.

For the current use of yuru-chara and the author's own primary research, the author is using two quantitative methods. This is because most current research into yuru-chara has either been reactive or quantitative. Firstly, the yuru-chara became popular, so then research has been done to find out why. Statistics such as sales are used to compare yuru-chara to other characters and marketing techniques. Other research has quantified characteristics, such as "humor" of yuru-chara to determine why a yuru-chara is successful or not (49TH: Regional Yuru-Chara Ranking 2017). While quantitative data are important, and need to be done, the author believes that qualitative data are also important for determining a yuru-chara's success and thus far research into this area appears to be lacking.

The two main research methods were interviews and observations. The interviews were mainly with company mascot character managers and interested fans of mascot characters. The observations mainly took place at the Yuru-Chara Grand Prix held in Matsuyama in November 2016. After initial observation for consistency, the participants and interviewees content will be analysed with brand marketing and identity theory from Paul Bloom (Bloom 2010). This is because yuru-chara do not have any functional use for a consumer to purchase an item with a yuru-chara on it. A brand and identity theory allows analysis on objects in the market that have no use or otherwise objective value.

\section{METHODOLOGY}

\section{What Are Yuru-Chara}

Yuru-chara are short for "yurui mascot characters", where yurui is a complicated Japanese word that can mean loose, easy, lazy, careless, halfhearted, or lenient. Japan has many cute, yurui characters to represent otherwise professional or serious groups, companies, or regions. Hikonyan and the thousands of others that soon followed, ushered in a new type of mascot, ones which were cute, lovable, and approachable. But most of all, these characters were yurui.

These mascot characters are everywhere, promoting cities, towns, municipalities, government agencies, and even companies. In August 2016, Tokyu Power Supply, an electric company, held a naming promotion over their new mascot character, an anthropomorphized light bulb (Tokyu Power Supply 2016).

These characters are mainly used for promotions, public relations, and branding. They appear on all manners of objects, especially near their hometowns. A couple of the yuru-chara have become so well-known and popular that you can purchase them nearly anywhere in Japan.

These yuru-chara are designed to be cute, but also designed to appeal to adults as well as children. For example, when the author visited Kaunet headquarters, she was introduced to Bouchan, an orange box with a smiling face that recently had become the new mascot. Bouchan does not fit the strict definition of yuru-chara, which is described below, because it does not hold special meaning to a region. Although it is not quite a yuru-chara, it is a representative of the style and it is a yurui mascot character. The team in charge of the Bouchan promotions described the character's design as being cute (kawaii), even though they had no intention of marketing him towards children. They mainly sold office supplies as a B-to-B business, and even their lesser direct to customer sales were mainly for office supplies. Their character design incorporated many ideas, but one of the main purposes of building the character and individual homepage was to create a space where the customers could "refresh" or feel “at ease" (Kaunet, personal communication, June 2, 2016). 
The team had not done in-depth market research into the viability of Kaubo. Instead, they used a naming competition to engage their customers in the new character and bring more recognition to the company. The character design was not an original concept, but instead was from a modification of the company logo. They wanted a character to compete with similar companies who already had designed a character, like Pencil Boy from ASKUL.

\section{History of Mascot Characters and Yuru-Chara}

However, Hikonyan was not the first yuru-chara. Mr. Miura coined the term to describe Bunkakki-, an oyster (kaki) character with a maple leaf hat, created for the Citizen's Prefectural Festival (kemmin bunkasai) in Hiroshima in 2000. At the time, yurui was used as derogatory as the creators did not put effort into creating the character. Later, Mr. Miura embraced the term, along with yuru-chara, as a positive character design.

When Mr. Miura coined the term, he also created the criteria for what makes a yuru-chara. Officially, yuru-chara must have three characteristics (Miura 2009):

(1) It must convey a strong message of love for one's hometown;

(2) The character's movements or behaviors should be unique and unstable or awkward;

(3) The character should be unsophisticated or laid-back and lovable.

In 2011, the first official, national Yuru-Chara Grand Prix was held, and every year since then, the number or participating yuru-chara has increased, and nearly doubling between 2012 and 2013. Some of these characters have become insanely popular within Japan (Yuru-Chara Grand Prix Official Website 2013).

While yuru-chara as a specific type of mascot characters are recent, mascot characters have been around for a long time, especially in athletics and children products. Internationally, the Olympics mascots have officially been around since 1972 with the dog Waldi for the Munich Olympics (Olympics 2016). Schools and universities often have a history of mascots, as well. American companies have had a rich history of "advertising characters" since the turn of the twentieth century (Dotz and Husain 2003).

In Japan, mascot characters have existed for more than half a century. In 1949, a frog character named Koro-chan was introduced for Kowa Company (Kowa Company, Ltd. 2008). In 1950, a dessert and pastry company called Fujiya introduced Peko-chan, a girl mascot character who constantly has her tongue sticking out (Fujiya Food Service Co. Ltd. 2007). Small, incremental introduction to mascot characters continued in Japan.

\section{Current Popularity}

Within Japan, mascot characters play a substantial role in marketing, branding, and marketing campaigns. Almost all major companies have mascot characters, such as Japan's National Broadcasting Corporation (NHK)'s Domo-kun (Japan Broadcasting Corporation 2011). Prefectures and regions also have mascot characters, which happen to be mostly yuru-chara. Many of these regional characters followed in the path of other popular characters such as Hello Kitty and have relatively easy obtainable licensing. Prefectural websites will typically have online application forms for permission to use the characters' image. Some yuru-chara like Kumamon, a bear character from Kumamoto Prefecture, can be used for free, even on commercial products.

Compared to the U.S., these characters are cuter, more popular, and more abundant. In a way, they are an untapped resource for the Cool Japan's marketing campaign abroad. There is an almost uncountable amount of yuru-chara in Japan. There is also the thought that there may be too many yuru-chara. Government offices in Osaka were asked to stop creating and even to cull some of the yuru-chara because it was starting to cause confusion and was 
perceived as excessive and a waste of money, as per an article in The Guardian (McCurry 2015).

One indication of how popular yuru-chara are becoming is the Yuru-Chara Grand Prix, which is held every year in a new city. In 2015, there were 1,727 yuru-chara registered for the event. That is nearly five times amount of entries compared to the first grand prix contest held in 2011 with 348 entries (Yuru-Chara Grand Prix 2016 Ranking 2016).

One of the most popular yuru-chara is the above-mentioned Kumamon. On April 20, a series of large earthquakes hit and devastated Kumamoto Prefecture and the surrounding areas. Agencies, donations, and relief workers have gathered around the symbol of Kumamoto to show support and raise funds and supplies to help the area. Before the event, Kumamon was already a popular character within Japan. He won the 2011 Grand Prix and has been on numerous television programs. Also, because he is free to license on most products, so you can find him on a variety of packaging, such as food, blankets, notebooks, and more. After the earthquake, Kumamoto Prefecture's government expedited the license application process for relief efforts because the process would take about a month (Kumamon Utilization Application 2017).

When visiting the Yuru-Chara Grand Prix in Matsuyama, the Japanese visitors interviewed there said that characters that they liked the most provided a sense of comfort and connection with their design. Many of the yuru-chara's motifs are taken from elements of the area or company (Yuru-Chara Grand Prix 2016 Ranking 2016). It provides a sense of connection to be community to those directly involved. At the Grand Prix, comfort, local pride, attractiveness, and authenticity seem to be the main elements that indicate successful yuru-chara.

While the characters typically do not speak, outside of a few exceptions, their "voice" when handling social media is crucial to this image. Using nyan as a cat and wan as a dog is so common that it would be odd if it were not the case. This means that the yuru-chara can give a face to the voices of the PR (public relation) arms of municipalities and companies. Yuru-chara "use” Twitter, Instagram, Facebook, along with websites, TV appearances, and in person appearances to promote their company or region. When before, the "town" itself, or perhaps a public worker, would send out the information. Now, the characters can communicate the desired information. It provides a personal connection to the audience that might not have been there before. At least that is the intent.

\section{Character Management and Brand Management}

Tribeck Brand Strategy Research Company analyzes yuru-chara with seven distinct characteristics: intimacy, personally, impact, humor, matches outsider image, holds local interests, and improves the impression of the area (Brand Strategy Communications 2011).

The author's argument is that beyond these seven characteristics, there is one more that is essential and is already woven into the current ones. It is the idea of identity, in so far as the consumer would purchase the product with the yuru-chara on it, because the yuru-chara reflect a part of the consumer. It is the idea that "I am a person who would like something like this, so I will buy it to show off my interests”. It is the theory as to why brands have become so popular.

This also explains why some yuru-chara, which have very off-putting characteristics, such as Chitchai-Ossan from Hyogo, have become controversial but popular (Upright Co. Ltd. 2014). It is the subversion itself that is popular. Yuru-chara already have one aspect that allows it inroad with consumer identity. Most yuru-chara are designed to be the mascot of a certain area. This allows the people in that area an instant connection with the yuru-chara because of local pride and tribalism. 
At the Yuru-Chara Grand Prix, many of the participants interviewed ranked the local to Matsuyama yuru-chara, Mican, as one of their favorites. However, Mican was not allowed to participate in the Grand Prix and could not win. Instead, it was considered one of the "special guest" yuru-chara, which also included past winners. Guests at the Grand Prix speculated that is because last year's winner was the local yuru-chara mascot of that region, Ieyasu-kun from Hamamatsu, and the event coordinators were trying to avoid that this year.

Thus, popular characters must also have good brand identity for the Japanese population beyond their local demographic characteristics. Characters such as Kumamon, Hikonyan, Gunma-chan, and Funassyi- are all able to ascend beyond into most of Japan. Consumers throughout Japan can identify with these characters. They score high on the seven characteristics above. Characters such as Sento-kun and Chitchai-Ossan also become more popular because they are subversive and not cute and there is a subversive brand identity existing alongside mainstream brand identity.

All these characters are also easily identifiable. Kumamon is a bear, Hikonyan is a cat, Gunma is a horse, and Chitchai-Ossan is human. Funassyi- might not be as easily identifiable by appearances along, but the "nashi" in his name helps Japanese consumers. And while a bit strange, Sento-kun is also identifiable as a humanoid, if not strictly human.

Distinct names are also essential, like Funassyi-, because the names are simple and usually a clue as to the character's identity. Kuma in Kumamon refers to both Kumamoto and bear. The nyan in Hikonyan refers to a cat. Additionally, for a yuru-chara to be successful as a yuru-chara and not just a mascot character, they must be simple and usually incorporate one or two aspects for which a person can identify with. The more aspects added to a character, the less people will identify with all the characteristics.

In contrast to the successful yuru-chara, including
Kumamon, Hikonyan, Gunma-chan, and Funassyi-, unsuccessful brand yuru-chara are too numerous to name. One reason why they are unsuccessful is that often they are too local or niche. Some designers try to put everything noteworthy about their area into one character. This leads to confusing looking characters, such as Chan-nishiki-chan from Nabari, Mie. A consumer cannot easily identify this yuru-chara from sight alone. Other characters, such as Ii-chan from Iijima in Nagano, are vague and unidentifiable. They are, in a sense, too generic to produce any brand identity. Ii-chan is a good name for the area, since it refers to Iijima. However, ii means cooked rice and the yuru-chara itself has nothing to do with rice, so it does not help identify the yuru-chara. Chan-nishiki-chan's name is long and confusing to anyone outside of Nabari.

\section{Japan Abroad}

Anime has been hugely successful abroad. Anne Allison in Millennial Monsters: Japanese Toys and the Global Imagination (Allison 2006), investigates Japanese toys, games, and anime in the U.S. and how its foreignness helps add to their appeal. She contends that the appeal of Japanese characters, or monsters, is that they are a mixture of both foreign and familiar. And it is that specific mix that helps make them popular globally. Some evidence can be found in the fact that Pokémon and recent PokémonGo, with their combination of recognizable and unrecognizable monsters as the stars, are globally recognized games.

Paul Bloom in How Pleasure Works (Bloom 2010), identifies that buying branded products is connected to identity and how we perceive ourselves and our own happiness. This means that authenticity of the product is crucial for the product's identity or brand to succeed. The mostly young people who purchase Cool Japan merchandise identify with Cool Japan and it is part of their identity. In the author's article published in Japan SPOTLIGHT (Suter 2015), the author argues that since yuru-chara have not been created with the even 
the slightest intent to introduce them to an international market, that their authenticity is higher than that of a product designed to appeal to both Japanese and international consumers.

Japan's Cool Japan Project is a government program aimed at capitalizing on the popularity boom of Japanese products such as anime characters outside Japan starting in the 1990s (Cool Japan/Creative Industries Policy 2013). One of their current missions is the "(e)nhancement of the Japanese Brand through Efforts to Open up and Expand Cool Japan Business Ventures”. However, while the successful projects are publicized for how well Japanese products do aboard, there are also examples of "Cool Japan" that have been unsuccessful. For example, there was the unsuccessful venture with the "Japanese High School Girls Ambassadors”.

Planning success does not represent such an insurmountable goal as one would be led to believe. A case can be made that the mystery certain characters and researching to find out what something means can be inciting as well. In 1996, NHK created a mascot character called Domo-kun (Walker 2007). While technically not a yuru-chara, Domo-kun fits most of the criteria that the yuru-chara meet for being interesting to international consumers. First, he was designed by someone Japanese for a local Japanese market. This makes him authentic. Second, he is cute, but also a bit slow and awkward. This is a relatively unusual and interesting combination in the U.S.

Domo-kun had received wide attention from international consumers, first on the Internet and then with actual physical products. In 2014, Domo-kun products, such as bags, hats, and shirts were still being sold in U.S. shops. He also became the mascot for 7-11 Slurpee ${ }^{\mathrm{TM}}$ in 2011 and Target Halloween collection in 2011, as well (Walker 2007).

\section{RESULTS AND DISCUSSION}

The observations in and of themselves are limited in scope because of the nature of the data collection. However, the implications and limitations will be discussed at length in the discussion section of this paper.

Yuru-chara appeal abroad would not be the same as their appeal in Japan. The author interviewed two international travelers interested in yuru-chara at the Yuru-Chara Grand Prix in Matsuyama on November 6, 2016. They mentioned that while they loved the yuru-chara, they could not imagine them being used as representatives or mascots in their home country of Canada because they do not convey enough authority (Anon, personal communication, November 6, 2016).

An American the author interviewed, Elizabeth Quart, who works part-time as a designer in Japan and has a working knowledge of both Japan and American culture, also agrees that yuru-chara "could (be popular in the U.S.) but it may be slow coming. There are mascots as it is in sports teams and even businesses, but rarely they are accepted as something more outside of that setting or targeted group” (E. M. Quart, personal communication, August 12, 2016).

It also has been mentioned that while yuru-chara can sell well and become popular, they are not merely representing themselves. They are, at the most basic level, mascot characters for a region, company, governmental office, or something similar. They were made to support something and were not created for their own sake. Their representation would be lost on people outside of Japan for the most part. For example, Kumamon is a bear because the kuma in Kumamoto means bear in Japanese. However, anyone who does not speak Japanese, most foreign consumers, would not be able to make this connection naturally and would have to be told.

It is important to keep in mind that while translations need be handled with care, it is even more important to consider pure look of the character and how that might translate across cultures. For example, in Japan, Kumamon's expression was explained as being full of wonder at the magnificent things around 
him in Kumamoto Prefecture (Mizuno 2012). However, while not exceeding popular in Japan, Kumamon has showed up on some English-speaking websites. A common image of Kumamon is him staring at the camera with a bonfire in the background containing the quote "Why? For the glory of Satan, of course". Additionally, his English consumer-given nick-name is "For the Glory of Satan Bear" (For the Glory of Satan Bear 2017). Obviously, his expression is seen a bit harsher in the U.S. than in Japan.

For further research, the author would like to conduct focus groups of American consumers with different amounts of details given about multiple characters to estimate how popular yuru-chara could become as a pure consumer item in the U.S., as opposed to a mascot item.

\section{CONCLUSIONS}

Yuru-chara and other mascot characters are hugely popular in Japan and could be a viable part of Cool Japan and Japanese brand identity expansion if used correctly. They are recognizable characters to the international market, but also different enough to be singled out as Japanese. Again, they are combining familiarity with foreignness that has worked well for the most popular Japanese products internationally. They have built in popularity and authenticity within Japan, both major components for brand awareness.

Beyond just marketing purposes, understanding why yuru-chara like Hikonyan are popular in Japan and not elsewhere provides valuable insight into the mindset of Japanese people and their marketing strategies. This provides the potential of using cute and kawaii to connect to the consumers abroad beyond children and people already interested in Cool Japan. And studying these characters more could provide a chance to close the gap between cultural misunderstandings with a thoughtful approach to global marketing solutions.

\section{Acknowledgments}

The author would like to thank her advisors, Takeo Kuwahara and David Freedman. Partial funding for this project was supported by the Ministry of Education, Culture, Sports, Science, and Technology of Japan, the Keio University Academic Development Funds for Individual Research.

\section{Note}

* The author declares no conflict of interest. The founding sponsors had no role in the design of the study; in the collection, analyses, or interpretation of data; in the writing of the manuscript; and in the decision to publish the results.

\section{References}

49TH: Regional Yuru-Chara Ranking. 2017. Retrieved (http://japanbrand.jp/ranking/nandemo/49.html).

Allison, A. 2006. Millennial Monsters: Japanese Toys and the Global Imagination. Berkeley: University of California Press.

Bloom, P. 2010. How Pleasure Works: The New Science of Why We Like What We Like. New York: W. W. Norton \& Company.

Brand Strategy Communications. 2011. 49TH: Regional Yuru-Chara Ranking. Retrieved March 15, 2017 (http://japanbrand.jp/ranking/nandemo/49.html).

Cool Japan/Creative Industries Policy. 2013. Retrieved (http://www.meti.go.jp/english/policy/mono_info_service/c reative_industries/creative_industries.html).

Dotz, W. and M. Husain. 2003. Meet Mr. Product: The Art of the Advertising Character. San Francisco: Chronicle Books.

For the Glory of Satan Bear. 2017. Retrieved (https://www. facebook.com/satanbeargonnagetyou).

Fujiya Food Service Co. Ltd. 2007. Peko-chan's Room. Retrieved March 20, 2017 (https://www.fujiya-peko.co.jp/ pekoroom/).

Japan Broadcasting Corporation. 2011. Domo-kun Museum. Retrieved March 20, 2017 (http://www.nhk.or.jp/domo/ index.html).

Kowa Company, Ltd. 2008. Kowa's Kerokoro Land. Retrieved March 20, 2017 (http://kr2.kowa.co.jp/history).

Kumamon Utilization Application. 2017. Retrieved (http:// kumamon-official.jp/application).

McCurry, J. 2015. “Character Assassination as Japan’s Mascot Ranks Are Trimmed.” The Guardian. Tokyo, April 9, 2015.

Miura, J. 2005. Yuru-Chara Illustrated Encyclopedia. Tokyo: Fusosha Publishing, Inc. 
2009. Recently, Have I Become a Yuru-Chara? ORICON. Retrieved March 20, 2017 (http://web.archive.org/web/ 20130515082549/http://www.oricon.co.jp/news/special/710 89/).

Mizuno, M. 2012. The Output Switch. Tokyo: Asahi Newspaper Publishing.

Olympics. 2016. Retrieved (http://www.olympics.com/ mascots).

Suter, R. 2015. "Yuru-Chara Have High Potential in International Markets.” Japan SPOTLIGHT July/August 2015(202):58-59.

Tokyu Power Supply. 2016. Retrieved (https://www.tokyu-ps. jp/campaign/namae.html).

Upright Co. Ltd. 2014. Chitchai-Ossan. Retrieved April 8, 2017 (http://co3.tv/).

Walker, R. 2007. Hello, Kitschy. Retrieved (http://www. nytimes.com/2007/07/22/magazine/22wwln-consumed-t.ht $\mathrm{ml}$ ?scp+1\&sq=domo\%20kun\&st_cse\&_r=0).

Yuru-Chara Grand Prix 2016 Ranking. 2016. Retrieved (http://www.yurugp.jp/vote/result_ranking.php).

Yuru-Chara Grand Prix Official Website. 2013. Retrieved March 20, 2017 (http://www.yurugp.jp/index.php).

Yuru-Chara Matsuri in Hakone Kigurumi Summit 2008. 2008. Retrieved March 20, 2017 (http://www.hikone-150th.jp/ event/contents/001114.php).

\section{Bio}

Jillian Rae Suter, M.A., Ph.D. candidate, Keio University, Japan; research fields: media and governance, marketing, sociology. 